\title{
Predictive factors of contralateral occult carcinoma in patients with papillary thyroid carcinoma: a retrospective study
}

\author{
Xiaohang Chen ${ }^{1,2 \#}$, Zhenwei Zhong ${ }^{1,2 \#}$, Muye Song ${ }^{1,3 \#}$, Jiru Yuan ${ }^{1,2}$, Ziyang Huang $^{1,2}$, Jialin Du ${ }^{1}$, \\ Yongchen Liu ${ }^{1}$, Zeyu $\mathrm{Wu}^{1}$ \\ ${ }^{1}$ Department of General Surgery, Guangdong Provincial People’s Hospital, Guangdong Academy of Medical Sciences, Guangzhou, China; ${ }^{2}$ Shantou \\ University Medical College, Shantou, China; ${ }^{3}$ South China University of Technology, Guangzhou, China \\ Contributions: (I) Conception and design: All authors; (II) Administrative support: Z Wu; (III) Provision of study materials or patients: Z Zhong, \\ M Song; (IV) Collection and assembly of data: J Yuan, Z Huang; (V) Data analysis and interpretation: X Chen, J Du; (VI) Manuscript writing: All \\ authors; (VII) Final approval of manuscript: All authors. \\ \#These authors contributed equally to this work. \\ Correspondence to: Yongchen Liu; Zeyu Wu. Department of General Surgery, Guangdong Provincial People's Hospital, Guangdong Academy of \\ Medical Sciences, 106 Zhong Shan Second Road, Guangzhou, China. Email: liuyongchen@gdph.org.cn; wu.zeyu@hotmail.com.
}

Background: The surgical approach toward unilateral papillary thyroid carcinoma (PTC) has been in controversy. One of the concerns is the existence of contralateral occult carcinoma, which could cause relapse and even lead to re-operation if not dealt with. This study aims to find out risk factors related to contralateral occult PTC, in order to facilitate in surgical approach decision for PTC.

Methods: A total of 921 PTC patients who underwent total/near-total thyroidectomy and central lymph node dissection (CND) with/without lateral lymph node dissection (LND) from January 2014 to Sept 2017 in Guangdong General Hospital were assessed retrospectively. The relations between contralateral occult PTC and clinicopathologic characteristics of PTC were analyzed by univariate and multivariate logistic regression.

Results: The incidence of contralateral occult carcinoma in patients with PTC was 16.7\% (154 of 921 cases). Univariate analysis showed that multifocality of the primary carcinoma $(\mathrm{P}=0.000)$, lymph node metastasis $(\mathrm{P}=0.001)$, pathologic tumor size $(\mathrm{P}=0.014)$ and contralateral benign nodule $(\mathrm{P}=0.000)$ were significantly associated with the increased incidence of contralateral occult PTC. No significant correlations were found between contralateral carcinoma and other variables such as gender $(\mathrm{P}=0.338)$, age $(\mathrm{P}=0.283)$, BRAF mutation $(\mathrm{P}=0.187)$ or extrathyroidal extension $(\mathrm{P}=0.423)$. Multivariate logistic regression analysis revealed that contralateral benign nodule $(\mathrm{P}=0.000)$, multifocality $(\mathrm{P}=0.000)$ and lymph node metastasis $(\mathrm{P}=0.009)$ were independent predictors of bilateral PTC of patients whose pre-operation ultrasound (US) show a unilateral carcinoma.

Conclusions: Lymph node metastasis, contralateral benign nodule and multifocality are independent predictors of contralateral occult PTC. For unilateral PTC patients with one or more of these factors, total/ near-total thyroidectomy should be considered when making surgical approach decisions.

Keywords: Contralateral occult carcinoma; lymph node metastasis (LNM); multifocality; papillary thyroid carcinoma (PTC)

Submitted Jun 13, 2019. Accepted for publication Jun 12, 2020.

doi: $10.21037 /$ gs-19-157

View this article at: http://dx.doi.org/10.21037/gs-19-157 


\section{Introduction}

The most widespread endocrine cancer is thyroid cancer. The overall thyroid cancer incidence increased from 4.9 to 14.7 per 100,000 people in 2011 (1). There are four main types of thyroid cancer, which includes papillary (PTC), follicular (FTC), anaplastic (ATC) and medullary thyroid cancer (MTC). The most common thyroid cancer type is PTC (2-5). PTC can not only occur as a single nodule, but also two or more anatomically separate foci in either unilaterally or bilaterally in the thyroid lobes (6-9).

Neck ultrasound (US) is a common way to detect PTC. High-resolution US can detect thyroid nodules in 19-68\% of individuals, of which $7-15 \%$ of these cases are thyroid cancer (10-12).

According to 2015 ATA guidelines, thyroid lobectomy can be a sufficient treatment for patients with PTC $<4 \mathrm{~cm}$, and without extrathyroidal extension or clinical evidence of lymph node metastases (13).

But is thyroid lobectomy sufficient for unilateral PTC? It has been reported that the rate of contralateral occult PTC discovered in total/subtotal thyroidectomy specimens was from $13-56 \%$ (14). Occult carcinoma is defined as a tumor focus in the contralateral lobe that is not detected preoperatively, but pathologically after surgery. If not dealt with, contralateral occult PTC could cause relapse and even lead to re-operation, which brings higher risks. But there haven't been enough studies and discussions of contralateral occult carcinoma. Thus there is lacking in evidence when making surgical approach decision for patients with unilateral PTC diagnosed by pre-operative examinations. With knowledge of such lack, our study aimed to find out risk factors related to contralateral occult PTC in patients with unilateral PTC that are confirmed by pre-operation examinations.

We present the following article in accordance with the STROBE guideline checklist (available at http://dx.doi. org/10.21037/gs-19-157).

\section{Methods}

We retrospectively reviewed medical records of 921 patients with PTC who underwent total/subtotal thyroidectomy and central lymph node dissection (CND) with/without lateral lymph node dissection (LND) from January 2014 to Sept 2017 in Guangdong Provincial People's Hospital. And pre-operation US showed unilateral PTC, which were performed by experienced sonologists in Guangdong Provincial People's Hospital.
We divided all patients into two groups according to post-operative pathological findings: patients with bilateral PTCs (154, 16.7\%), which meant contralateral occult PCT was confirmed and those with unilateral PTCs (767, $83.3 \%)$. Bilateral PTC is defined as cancer diagnosed histopathologically in both thyroid lobes at the same time. Patients who had a solitary tumor focus or multiple tumor foci located in one thyroid lobe are classified as having unilateral PTC. And the maximum of diameters of multiple tumor foci was taken. Clinicopathologic and demographics characteristics, including age, gender, tumor size, BRAFv600E mutation, multifocality of the primary carcinoma and lymph node metastases, were retrospectively reviewed and analyzed. There were 643 females (69.8\%) and 278 males (31.2\%), ranging in age from 13 to 77 years, with a mean age of $42.7 \pm 12.5$ years. Tumor diameter was $>1 \mathrm{~cm}$ on ultrasonography in 509 cases $(55.3 \%)$, while tumor diameter was $\leq 1 \mathrm{~cm}$ in 412 cases (44.7\%). And the mean tumor diameter was $1.46 \pm 0.89 \mathrm{~cm}$. The number of patients with benign nodule in the contralateral lobe showed in pre-operation US was 347 (37.7\%). Pathological observations revealed 224 tumors (24.3\%) with extrathyroidal extension (ETE). And lymph node metastasis was found in 445 patients (48.3\%). Pathological observations found out that there were 77 multifocal tumors $(8.4 \%)$ of the primary carcinoma. The histological diagnosis was classified according to the World Health Organization system (15). The association between contralateral occult PTC and clinicopathological features of PTC was analyzed in this study.

\section{Statistical analysis}

The date collection was completed with Microsoft Excel. The clinicopathological features among groups were compared using the Pearson chi square test. Multivariate analysis was performed by binary logistic regression. $\mathrm{P}$ value $<0.05$ was considered statistically significant. All analyses were performed with the Statistical Package for Social Sciences (SPSS, Inc., Chicago, IL, USA) for Windows.

\section{Results}

In this study, the incidence of contralateral occult PTC in patients was $16.7 \%$ (154 cases). There were $37.7 \%$ (347 cases) of patients have contralateral benign nodule of thyroid showed in pre-operation US. There were 445 (48.3\%) patients with lymph node metastases, and 224 
(24.3\%) with extrathyroidal extension. And 328 (35.6\%) patients were found to have BRAFv600E mutation positivity confirmed post-operatively.

\section{Correlations between bilateral PTC and clinicopathological characteristics of PTC}

Univariate analysis showed that bilateral PTC of patients whose pre-operation US showed a unilateral PTC was significantly associated with tumor size, existence of contralateral nodule and lymph node metastases. In 347 patients with contralateral benign nodule, 93 (26.8\%) cases were confirmed as contralateral occult PTC. While in 574 patients without contralateral benign nodule, only $61(10.6 \%)$ cases came out to be bilateral PTC. The difference between these two groups was statistically significant $(\mathrm{P}=0.00)$. Bilateral PTC was more frequently found in patients with tumor size $>1 \mathrm{~cm}$ (99 of 509 cases, $19.4 \%)$, compared with patients whose tumor size $\leq 1 \mathrm{~cm}$ (55 of 412 cases, $13.3 \%)(\mathrm{P}=0.014)$. The incidence rate of bilateral PTC in patients with lymph node metastases was $20.8 \%$ (93 of 455 cases), whereas it was only $12.8 \%$ (61 of 476 cases) in patients without lymph node metastases. And the difference between these two groups showed statistical significance $(\mathrm{P}=0.001)$. Contralateral occult PTC appeared more frequently in patients with multifocality of the primary carcinoma (33 of 77 cases, $42.9 \%$ ), compared to patients without multifocality (121 of 844 cases, $14.3 \%$ ) $(\mathrm{P}=0.000)$. No significant correlations were found between bilateral PTC and other variables such as gender $(\mathrm{P}=0.338)$, age $(\mathrm{P}=0.283)$, extrathyroidal extension $(\mathrm{P}=0.423)$ and BRAFv600E mutation $(\mathrm{P}=0.187)$ (Table 1). Multivariate logistic regression analysis revealed that contralateral benign nodule $(\mathrm{P}=0.000)$, multifocality $(\mathrm{P}=0.000)$ and lymph node metastasis $(\mathrm{P}=0.009)$ were independent predictors of bilateral PTC of the patients whose pre-operation US showed a unilateral PTC (Table 2).

\section{Discussion}

The incidence of PTC has increased rapidly in recent decades due to improved diagnostic accuracy of US, fine needle aspiration biopsy (FNAB) and increased awareness of thyroid nodular disease (16). Total or subtotal thyroidectomy, thyroid lobectomy, TSH suppression and radioiodine ablation have been universally accepted as standard treatments for thyroid cancer. According to the 2015 ATA guideline and a guide to diagnosis and treatment of differentiated thyroid cancer of China Anticancer Association head and neck tumor Specialized Committee (17), thyroid lobectomy could be a sufficient treatment for patients with PTC $>1$ and $<4 \mathrm{~cm}$, without extrathyroidal extension or clinical evidence of lymph node metastases (13). But when unilateral lobectomy is performed for patients with contralateral occult PTC, the occult PTC would cause recurrence and even require reoperation, which is related to higher surgical risks compared with primary surgery. The study of Ritter $e t a l$. reported that in 167 patients of unilateral PTC who underwent lobectomy, during a mean follow-up of 6.5 years, 18 patients (10.7\%) had completion thyroidectomy, among which 12 of them were diagnosed as contralateral PTC $(\mathrm{n}=8,4.8 \%)$ or lymph node metastasis ( $n=4,2.4 \%)$ (18). According to Unalp, H.R, re-operation increased the incidence rate of complications including hypoparathyroidism and recurrent laryngeal nerve paralysis by 8 to 27 times (19), compared to that of primary surgery. Bilimoria et al. (20) reported a slightly lower 10-year recurrence rate for patients who received a total/subtotal thyroidectomy (total/subtotal thyroidectomy $7.7 \%$ vs. unilateral lobectomy $9.8 \%$, respectively, $\mathrm{P}<0.05$ ), which meant a lower re-operation rate (20). But there are different findings. Haigh et al. analyzed 4,612 patients receiving total thyroidectomy and 820 patients receiving lobectomy and found that there was no difference in 10 -year overall survival and 10-year recurrence rate between total thyroidectomy and thyroid lobectomy groups (21). Considering this controversy, plus the fact that most PTCs showed excellent prognosis, surgical extent of unilateral PTC diagnosed pre-operatively should be well discussed and carefully decided.

It is very common that well-differentiated thyroid cancer is accompanied by cervical lymph node metastases. Lymph node metastasis has been reported to be associated with increased rates of recurrence and worse overall survival of patients with thyroid cancers (22). In our study, lymph node metastases were found in $18.7 \%$ (72 in 386 cases) patients with contralateral occult PTC $(\mathrm{P}=0.004)$. Multivariate analysis showed that lymph node metastasis was an independent predictor of contralateral occult PTC for patients whose pre-operation US showed a unilateral PTC. Several recent studies reported that lymph node metastasis is a predictive factor of bilateral PTC. Theodore Karatzas et al. reported that in 34 PTC patients with lymph node metastasis, 21 patients $(61.8 \%)$ had contralateral PTC; while only 56 patients (15.3\%) had contralateral PTC in 385 patients without lymph node metastasis $(\mathrm{P}<0.001)$. Pacini et al. also reported similar result $(23,24)$. 
Table 1 Univariate analysis of the correlations between contralateral lesion and clinicopathologic characteristics of PTC

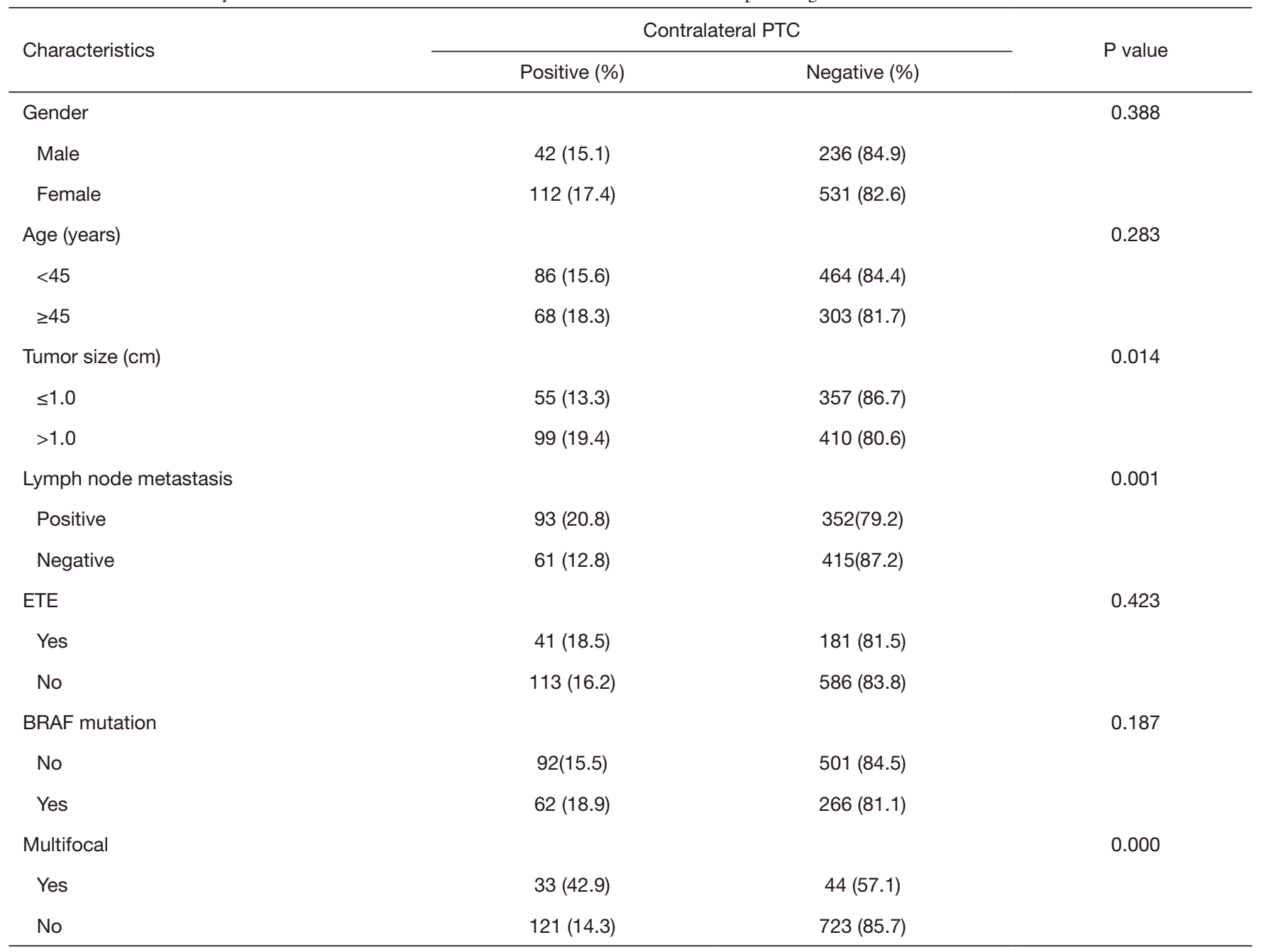

PTC, papillary thyroid carcinoma; ETE, extrathyroid extension.

Table 2 Multivariate analysis of the correlations between contralateral lesion and clinicopathologic characteristics of PTC

\begin{tabular}{|c|c|c|c|c|c|c|c|c|}
\hline \multirow{2}{*}{ Characteristics } & \multirow{2}{*}{$\mathrm{B}$} & \multirow{2}{*}{ S.E. } & \multirow{2}{*}{ Wals } & \multirow{2}{*}{$\mathrm{df}$} & \multirow{2}{*}{ Sig. } & \multirow{2}{*}{$\operatorname{Exp}(B)$} & \multicolumn{2}{|c|}{$95 \% \mathrm{Cl}$} \\
\hline & & & & & & & Lower & Upper \\
\hline Tumor size & 0.309 & 0.192 & 2.587 & 1 & 0.108 & 1.363 & 0.935 & 1.983 \\
\hline $\begin{array}{l}\text { Lymph node } \\
\text { metastasis }\end{array}$ & 0.415 & 0.189 & 4.811 & 1 & 0.028 & 1.514 & 1.045 & 2.192 \\
\hline Multifocality & 1.404 & 0.254 & 30.648 & 1 & 0.000 & 4.071 & 2.477 & 6.692 \\
\hline
\end{tabular}

PTC, papillary thyroid carcinoma. 
Previous studies reported that tumor size had been proven to be independent predictors for bilaterality in PTC patients $(25,26)$. Hwang et al. also reported that bilaterality was more commonly found in patients with advanced $\mathrm{T}$ stage (27). However, Mazeh et al. reported a different result. They divided 263 patients into five groups according to the tumor size [group 1, 1 to $10 \mathrm{~mm}$ (microcarcinoma) in $60(23 \%)$ patients; group 2,11 to $20 \mathrm{~mm}$ in $110(42 \%)$ patients; group 3, 21 to $30 \mathrm{~mm}$ in 44 (17\%) patients; group 4, 31 to $40 \mathrm{~mm}$ in $30(11 \%)$ patients; and group 5, larger than $40 \mathrm{~mm}$ in $19(7 \%)$ patients]. $57 \%$ of patients in group 1,2 and 4 had contralateral PTC; $61 \%$ of patients in group 3 had contralateral PTC; $47 \%$ of patients in group 5 had contralateral PTC $(\mathrm{P}=0.6)$ (28). In our study, multivariate analysis showed that tumor size $>1 \mathrm{~cm}$ was an independent predictor of contralateral occult PTC in patients whose pre-operation US showed a unilateral PTC. The difference in size cut-offs in each of those studies might affect the association between the incidence of bilateralilty and tumor size. Further randomized controlled multicenter study would be helpful to draw a more precise conclusion.

In this study, contralateral nodule was associated with bilaterality in PTC patients with pre-operation US showing a unilateral PTC $(\mathrm{P}<0.001)$. Multivariate analysis showed contralateral nodule was an independent predictor of contralateral occult PTC in such patients $(\mathrm{P}=0.000)$. Koo reported that contralateral occult PTC were found in $24.3 \%$ patients who had contralateral benign nodule (18 of 74 cases), which was significantly different compared with $6.9 \%$ patients without contralateral benign nodule ( 4 of 58 cases; $\mathrm{P}=0.009$ ), leading to similar result (14). Lee et al. also found existence of nodule in contralateral lobe related to contralateral occult PTC (29). Nevertheless, it is possible that some of the contralateral tumor foci were misdiagnoses as benign nodules during pre-operation examination, which could cause some biases of the result. Also, lack of FNAB of contralateral nodules could possibly lead to partial inaccuracy of this study.

Previous studies found out that ipsilateral multifocality was a clinicopathological feature predictive of contralateral occult PTC, similar to finding of this study. Kim et al. reported that in 672 PTC patients with ipsilateral multifocality, 59 patients $(8.8 \%)$ had local recurrence; while there were only 79 patients $(5.6 \%)$ with local recurrence in 1,423 patients without ipsilateral multifocality $(\mathrm{P}=0.04)(30)$. Theodore Karatzas et al. also reported $66.0 \%$ patients with multifocality of primary carcinoma (68 of 103 cases) had contralateral occult PTC, while there were only $4.1 \%$ patients without multifocality of primary carcinoma ( 9 of 216 cases) had contralateral occult PTC $(\mathrm{P}<0.001)(23)$. In this study, multifocality of the primary carcinoma is a predictive factor of contralateral PTC.

BRAF mutation is the most potent activator of the mitogen-activated protein kinase pathway, and plays a central role in the regulation of cell growth, division and proliferation (31). Previous study reported that prevalence of BRAF mutations in PTC ranges from 29\% to $83 \%$ (32). Lee et al. reported that $31.2 \%$ patients with positive BRAF mutation had bilateral PTC. While in comparison, only $23.0 \%$ patients with negative BRAF mutation $(\mathrm{P}<0.001)(25)$ showed bilaterality. And study of Lv et al. also showed similar result (33). But other author had different findings. Lee et al. reported a different result, in which among 40 cases of post-operative diagnosed contralateral occult PTC, 29 cases $(72.5 \%)$ had positive BRAF mutation; compared to the fact that 82 in 128 cases $(64.0 \%)$ without contralateral PTC showed BRAF mutation positivity. And they drew a conclusion that BRAF mutation was not significantly related with contralateral occult PTC (34). Whether BRAF mutation is associated with contralateral PTC is still controversial. It needs more detailed sample selection to find out the relationship between BRAF mutation and contralateral occult PTC.

In this study, ETE appeared to be an independent predictive factor of contralateral occult PTC in patients pre-operatively diagnosed as unilateral PTC. While Hwang et al. reported that bilaterality were more commonly found in PTC patients with ETE (23.6\% with ETE vs. 9.7\% without ETE) (27). And Kim et al. reported a similar result when analyzing PTMC (35). The difference between result of this study and those of Hwang's and Kim's could be due to the different selection of samples, where the latter studies include pre-operatively diagnosed bilateral PTC. In addition, McConahey et al. reported that ETE increased the risk of PTC recurrence and mortality (36). So when it comes to contralateral PTC with ETE, total/near-total thyroidectomy should be performed.

\section{Conclusions}

When pre-operative US shows a unilateral PTC, contralateral occult lesions could be missed or misdiagnosed as benign nodules, which might lead to serial consequences including tumor recurrence, re-operation and heavier financial and mental stress for patients. Thus, decision on surgical approach toward preoperatively diagnosed unilateral 
PTC should be carefully considered and wisely chosen. As shown in this study, lymph node metastasis, existence of contralateral benign nodule, multifocality of primary carcinoma and larger tumor size are independent predictive factors of contralateral occult PTC. In conclusion, we suggest that when dealing with unilateral PTC with one or more of these features, surgeons should take total/near-total thyroidectomy into consideration.

\section{Acknowledgments}

Funding: Project supported by the Natural Science Foundation of Guangdong Province, China (No. 2020A1515010127); Project of Outstanding Young Medical Professionals of Guangdong Province, China (No. KJ012019441) and Initiation Project of Returned Overseas Students of Guangdong Provincial People's Hospital (No. 20171x02).

\section{Footnote}

Reporting Checklist: The authors have completed the STROBE guideline checklist. Available at http://dx.doi. org/10.21037/gs-19-157

Data Sharing Statement: Available at available at http:// dx.doi.org/10.21037/gs-19-157

Conflicts of Interest: All authors have completed the ICMJE uniform disclosure form (available at http://dx.doi. org/10.21037/gs-19-157). The authors have no conflicts of interest to declare.

Ethical Statement: The authors are accountable for all aspects of the work in ensuring that questions related to the accuracy or integrity of any part of the work are appropriately investigated and resolved. The study was conducted in accordance with the Declaration of Helsinki (as revised in 2013). The study was approved by Ethics Committee of the Guangdong Provincial People's Hospital (approval ID: 2019774H) and written informed consent was obtained from all patients.

Open Access Statement: This is an Open Access article distributed in accordance with the Creative Commons Attribution-NonCommercial-NoDerivs 4.0 International License (CC BY-NC-ND 4.0), which permits the noncommercial replication and distribution of the article with the strict proviso that no changes or edits are made and the original work is properly cited (including links to both the formal publication through the relevant DOI and the license). See: https://creativecommons.org/licenses/by-nc-nd/4.0/.

\section{References}

1. Davies L, Morris Luc GT, Haymart M, et al. American Association of Clinical Endocrinologists and American College of Endocrinology disease state clinical review: the increasing incidence of thyroid cancer. Endocr Pract 2015;21:686-96.

2. Giusti F, Falchetti A, Franceschelli F, et al. Thyroid cancer: Current molecular perspectives. J Oncol 2010;2010:351679.

3. Braun J, Hüttelmaier S. Pathogenic mechanisms of deregulated microRNA expression in thyroid carcinomas of follicular origin. Thyroid Res 2011;4 Suppl 1:S1.

4. Leonardi GC, Candido S, Carbone M, et al. MicroRNAs and thyroid cancer: Biological and clinical significance (Review). Int J Mol Med 2012;30:991-9.

5. Benvenga S, Koch CA. Molecular pathways associated with aggressiveness of papillary thyroid cancer. Curr Genomics 2014;15:162-70.

6. Kuhn E, Teller L, Piana S, et al. Different clonal origin of bilateral papillary thyroid carcinoma, with a review of the literature. Endocr Pathol 2012;23:101-7.

7. Carcangiu ML, Zampi G, Pupi A, et al. Papillary carcinoma of the thyroid-A clinicopathologic study of 247 cases treated at the University of Florence, Italy. Cancer 1985;55:805-28.

8. Katoh R, Sasaki J, Kurihara H, et al. Multiple thyroid involvement (intraglandular metastasis) in papillary thyroid carcinoma: a clinicopathologic study of 105 consecutive patients. Cancer 1992;70:1585-90.

9. Mazzaferri EL, Jhiang SM. Long-term impact of initial surgical and medical therapy on papillary and follicular thyroid cancer. Am J Med 1994;97:418-28.

10. Hegedüs L. Clinical practice. The thyroid nodule. N Engl J Med 2004;351:1764-71.

11. Mandel SJ. A 64-year-old woman with a thyroid nodule. JAMA 2004;292:2632-42.

12. Sherman SI. Thyroid carcinoma. Lancet 2003;361:501-11.

13. Haugen BR, Alexander EK, Bible KC, et al. 2015 American Thyroid Association Management Guidelines for Adult Patients with Thyroid Nodules and Differentiated Thyroid Cancer: The American Thyroid Association Guidelines Task Force on Thyroid Nodules and Differentiated Thyroid 
Cancer. Thyroid 2016;26:1-133.

14. Koo BS, Lim HS. Occult contralateral carcinoma in patients with unilateral papillary thyroid microcarcinoma. Ann Surg Oncol 2010;17:1101-5.

15. Hedinger C, Williams ED, Sobin LH. The WHO Histological Classification of Thyroid Tumors: a commentary on the second edition. Cancer 1989;63:908-11.

16. Karatzas T, Vasileiadis I, Kapetanakis S, et al. Risk factors contributing to the difference in prognosis for papillary versus micro-papillary thyroid carcinoma. Am J Surg 2013;206:586-93.

17. China Anticancer Association head and neck tumor Specialized Committee. A guide to diagnosis and treatment of differentiated thyroid cancer. Chinese Journal of Practical Surgery 2011. doi: 10.3969/ j.issn.1674-0904.2011.01.017.

18. Ritter A, Mizrachi A, Bachar G, et al. Detecting Recurrence Following Lobectomy for Thyroid Cancer: Role of Thyroglobulin and Thyroglobulin Antibodies. J Clin Endocrinol Metab 2020;105:dgaa152.

19. Unalp HR, Erbil Y, Akguner T, et al. Does near total thyroidectomy offer advantage over total thyroidectomy in terms of postoperative hypocalcemia? Int J Surg 2009; 7:120-5.

20. Bilimoria KY, Bentrem DJ, Ko CY, et al. Extent of surgery affects survival for papillary thyroid cancer. Ann Surg 2007;246:375-81.

21. Haigh PI, Urbach DR, Rotstein LE. Extent of thyroidectomy is not a major determinant of survival in lower high-risk papillary thyroid cancer. Ann Surg Oncol 2005;12:81-9.

22. Liu FH, Kuo SF, Hsueh C et al. Postoperative recurrence of papillary thyroid carcinoma with lymph node metastasis. J Surg Oncol 2015;112:149-54.

23. Karatzas T, Vasileiadis I, Charitoudis G, et al. Bilateral versus unilateral papillary thyroid microcarcinoma: predictive factors and associated histopathological findings following total thyroidectomy. Hormones (Athens) 2013;12:529-36.

24. Pacini F, Elisei R, Capezzone M, et al. Contralateral papillary thyroid cancer is frequent at completion thyroidectomy with no difference in low- and high-risk patients. Thyroid 2001;11:877-81.

25. Lee KJ, Cho YJ, Kim JG, et al. How many contralateral papillary thyroid carcinomas can be missed? World J Surg 2013;37:780-5.

26. Zhou YL, Gao EL, Zhang W, et al. Factors predictive of papillary thyroid micro-carcinoma with bilateral involvement and central lymph node metastasis: a retrospective study. World J Surg Oncol 2012;10:67.

27. Hwang E, Pakdaman MN, Tamilia M, et al. Bilateral papillary thyroid cancer and associated histopathologic findings. J Otolaryngol Head Neck Surg 2010;39:284-7

28. Mazeh H, Samet Y, Hochstein D, et al. Multifocality in well-differentiated thyroid carcinomas calls for total thyroidectomy. Am J Surg 2011;201:770-5.

29. Lee YC, Eun YG, Sohn YM, et al. Predictive factors for occult contralateral carcinoma in patients with unilateral papillary thyroid microcarcinoma by preoperative ultrasonographic and pathological features. World J Surg 2015;39:1736-41.

30. Kim HJ, Sohn SY, Jang HW, et al. Multifocality, but not bilaterality, is a predictor of disease recurrence/ persistence of papillary thyroid carcinoma. World J Surg 2013;37:376-84.

31. Mercer KE, Pritchard CA. Raf proteins and cancer: B-Raf is identified as a mutational target. Biochim Biophys Acta 2003;1653:25-40.

32. Xing M. BRAF mutation in thyroid cancer. Endocr Relat Cancer 2005;12:245-62.

33. Lv T, Zhu C, Di Z. Risk factors stratifying malignancy of nodules in contralateral thyroid lobe in patients with preoperative ultrasound indicated unilateral papillary thyroid carcinoma: A retrospective analysis from single centre. Clin Endocrinol (Oxf) 2018;88:279-84.

34. Lee YC, Eun YG, Sohn YM. Predictive factors for occult contralateral carcinoma in patients with unilateral papillary thyroid microcarcinoma by preoperative ultrasonographic and pathological features. World J Surg 2015;39:1736-41.

35. Kim SK, Park I, Woo JW, et al. Predicting factors for bilaterality in papillary thyroid carcinoma with tumor size $<4$ cm. Thyroid 2017;27:207-14.

36. McConahey WM, Hay ID, Woolner LB, et al. Papillary thyroid cancer treated at the Mayo Clinic, 1946 through 1970: initial manifestations, pathologic findings, therapy, and outcome. Mayo Clin Proc 1986;61:978-96.

Cite this article as: Chen $\mathrm{X}$, Zhong Z, Song M, Yuan J, Huang Z, Du J, Liu Y, Wu Z. Predictive factors of contralateral occult carcinoma in patients with papillary thyroid carcinoma: a retrospective study. Gland Surg 2020;9(4):872-878. doi: 10.21037/ gs-19-157 